\title{
ABOUT ONE INVERSE PROBLEM OF THE LINEAR-FRACTIONAL PROGRAMMING ON GENERALIZED NETWORK
}

\author{
L.A. Pilipchuk ${ }^{1 \S}$, A.S. Pilipchuk ${ }^{2}$ \\ Belarusian State University \\ Nezavisimosti avenue 4, Minsk, 220030, BELARUS
}

\begin{abstract}
For the one linear-fractional network programming problem with additional constraints of general kind and with inexact data we constructed a mathematical model for calculation of the new parameters of the restrictions for which the infeasible solution becomes feasible solution. For the selected feasible solution of this problem we minimally changed the parameters in the numerator of the linear-fractional objective function in order that the selected feasible solution has become an optimal. The measure proximity vectors estimated are using the norm $l_{1}$. That has allowed to remain within the framework of linear programming in solving of inverse problems.
\end{abstract}

AMS Subject Classification: 65K05, 90C08, 90C35, 05C50, 15A03, 15A06

Key Words: linear-fractional programming problem, inexact data, infeasible solution, feasible solution, duality, norm, inverse optimization, the numerator of linear-fractional objective function, optimal solution

\section{Mathematical Model}

Let $G=(I, U)$ be a finite oriented connected graph (network) without multiple arcs and loops, where $I$ is a set of nodes and $U \subset I \times I$ is a set of arcs. On

Received:

Revised: July 2, 2016

Published: September 15, 2016

September 30, 2016

${ }^{\S}$ Correspondence author (c) 2016 Academic Publications, Ltd. url: www.acadpubl.eu 
the described graph $G$ we consider the linear-fractional homogeneous flow programming optimization problem (1)-(4) with additional constraints of general kind:

$$
\begin{gathered}
f(x)=\frac{\sum_{(i, j) \in U} c_{i j} x_{i j}+\beta}{\sum_{(i, j) \in U} q_{i j} x_{i j}+\gamma} \longrightarrow \min , \\
\sum_{j \in I_{i}^{+}(U)} x_{i j}-\sum_{j \in I_{i}^{-}(U)} \mu_{j i} x_{j i}=a_{i}, \quad i \in I, \\
\sum_{(i, j) \in U} \lambda_{i j}^{p} x_{i j}=\alpha_{p}, \quad p=\overline{1, l}, \\
x_{i j} \geq 0, \quad(i, j) \in U,
\end{gathered}
$$

where $x_{i j}$ - an arc flow of $\operatorname{arc}(i, j) \in U, x=\left(x_{i j},(i, j) \in U\right)$ - arc flows vector of the graph $G=(I, U), I^{+}(U)=\{j \in I:(i, j) \in U\}, I_{i}^{-}(U)=\{j \in I:(j, i) \in$ $U\}, \mu=\left(\mu_{i j},(i, j) \in U\right)$ - vector of coefficients transformation of arc flows, $\mu_{i j}$ - the transformation coefficient of the arc flow $x_{i j}$ for $\operatorname{arc}(i, j)$ : arc flow $x_{i j}$ outgoing from the node $i$ and incoming to the node $j$ in a modified form $\mu_{i j} x_{i j}$, and, transformation arc flow $x_{i j}$ is carried out immediately before the node $j$, $\left.\left.\mu_{i j} \in\right] 0,1\right]$. Here $c_{i j}, q_{i j}, \mu_{i j}, a_{i}, \lambda_{i j}^{p}, \alpha_{p}, \beta, \gamma-$ known parameters of problem (1)-(4).

Let us assume, that the denominator

$$
q(x)=\sum_{(i, j) \in U} q_{i j} x_{i j}+\gamma
$$

of the objective function $f(x)$ does not change a sign for the set of flows $x \in X$. Without restriction of a generality, we shall assume that $q(x)>0, \forall x \in X$.

In practice, there are many situations when the values of the parameters are inexact data. For the first time the inverse optimization problems were considered in [1], [2] for changing the coefficients of the objective function for some special problems of linear programming. Let $x=\left(x_{i j},(i, j) \in U\right)$, $x_{i j} \geq 0,(i, j) \in U$ - basic infeasible solution extremal problem (1)-(4) for the given parameters (some constraints $(2)-(3)$ are not met). We will change the parameters of restrictions (2)-(3) for the problem (1)-(4) so that all restrictions are performed (basic feasible solution). 


\section{Adjustment of Parameters Constraints}

Consider the constraints of the problem (1)-(4). The following parameters

$$
a_{i}, i \in I ; \quad \alpha_{p}, p=\overline{1, l} ; \mu_{i j},(i, j) \in U ; \lambda_{i j}^{p}, p=\overline{1, l},(i, j) \in U
$$

are inexact data. It is necessary minimally to change the parameters (5) so that a given infeasible basic solution $x$ becomes feasible basic solution for new parameters, $X$ is the set of the feasible basic solutions. To change the parameters in the network optimization problems in [3], [4] were considered some mathematical models in accordance with the $l_{1}$. The selected norm $l_{1}$ allows to remain within the framework of linear programming.

When adjusting the parameters (5) of restrictions (2)-(3), the following two cases can be presented.

In the case 1) if the transform coefficients $\mu_{i j},(i, j) \in U$ of arc flows $x_{i j},(i, j) \in U$ and the matrix of coefficients of additional constraints (3) do not change, then the adjusted parameters $\tilde{a}_{i}, i \in I$ and $\tilde{\alpha}_{p}, \quad p=\overline{1, l}$ of problem (1)-(4) are computed as follows:

$$
\begin{gathered}
\tilde{a}_{i}=\sum_{j \in I_{i}^{+}(U)} x_{i j}-\sum_{j \in I_{i}^{-}(U)} \mu_{j i} x_{j i}, \quad i \in I, \\
\tilde{\alpha}_{p}=\sum_{(i, j) \in U} \lambda_{i j}^{p} x_{i j}, \quad p=\overline{1, l} .
\end{gathered}
$$

In the case 2) when change the parameters $a_{i}, i \in I ; \alpha_{p}, p=\overline{1, l}$ of boundaries for restrictions (2)-(3) and also the transform coefficients $\mu_{i j},(i, j) \in U$ of the arc flows $x_{i j},(i, j) \in U$ and the matrix of coefficients of additional constraints (3), the new parameters have the following form:

$$
\begin{gathered}
\widetilde{a}_{i}=a_{i}+k_{i}-\psi_{i}, k_{i} \geq 0, \psi_{i} \geq 0, \quad i \in I \\
\widetilde{\alpha}_{p}=\alpha_{p}+\varphi_{p}-\delta_{p}, \varphi_{p} \geq 0, \delta_{p} \geq 0, p=\overline{1, l} \\
\widetilde{\mu}_{i j}=\mu_{i j}+\theta_{i j}-\gamma_{i j}, \theta_{i j} \geq 0, \gamma_{i j} \geq 0, \quad(i, j) \in U, \\
\widetilde{\lambda}_{i j}^{p}=\lambda_{i j}^{p}+r_{i j}^{p}-t_{i j}^{p}, p=\overline{1, l}, \quad(i, j) \in U .
\end{gathered}
$$

The values of increase and decrease for the parameters (5) are defined as follows:

- $k_{i}$ - increase and $\psi_{i}$ - decrease of parameter $a_{i}$;

- $\varphi_{p}$ - increase and $\delta_{p}$ - decrease of parameter $\alpha_{p}$; 
- $\theta_{i j}$ - increase and $\gamma_{i j}$ - decrease of parameter $\mu_{i j}$;

- $r_{i j}^{p}$ - increase and $t_{i j}^{p}$ - decrease of parameter $\lambda_{i j}^{p}$.

It is necessary to find such new parameter (6) that the infeasible basic solution $x=\left(x_{i j},(i, j) \in U\right)$ of the problem (1)-(4) for the parameters (5) was a feasible basic solution for the new parameters (6) of the extremal problem of the following form:

$$
\begin{gathered}
f(x)=\frac{\sum_{(i, j) \in U} c_{i j} x_{i j}+\beta}{\sum_{(i, j) \in U} q_{i j} x_{i j}+\gamma} \longrightarrow \min , \\
\sum_{j \in I_{i}^{+}(U)} x_{i j}-\sum_{j \in I_{i}^{-}(U)} \tilde{\mu}_{j i} x_{j i}=\tilde{a}_{i}, \quad i \in I, \\
0<\tilde{\mu}_{i j} \leq 1, \quad(i, j) \in U, \\
\sum_{(i, j) \in U} \tilde{\lambda}_{i j}^{p} x_{i j}=\tilde{\alpha}_{p}, \quad p=\overline{1, l}, \\
x_{i j} \geq 0, \quad(i, j) \in U .
\end{gathered}
$$

Let us note that the total parameter adjustments is a minimum in accordance with the selected norm $l_{p}$ for the sum of vectors:

$$
\begin{gathered}
l_{p}=\|\tilde{a}-a\|+\|\tilde{\alpha}-\alpha\|+\|\tilde{\mu}-\mu\|+\|\tilde{\lambda}-\lambda\|, \\
a=\left(a_{i}, i \in I\right), \tilde{a}=\left(\tilde{a}_{i}, i \in I\right), \\
\alpha=\left(\alpha_{p}, p=\overline{1, l}\right), \tilde{\alpha}=\left(\tilde{\alpha}_{p}, p=\overline{1, l}\right), \\
\mu=\left(\mu_{i j},(i, j) \in U\right), \tilde{\mu}=\left(\tilde{\mu}_{i j},(i, j) \in U\right) . \\
\lambda=\left(\lambda_{i j}^{p}, p=\overline{1, l},(i, j) \in U\right), \tilde{\lambda}=\left(\tilde{\lambda}_{i j}^{p}, p=\overline{1, l},(i, j) \in U\right) .
\end{gathered}
$$

Given that the increasing and decreasing for the value of each parameter is not may be simultaneously positive and using (6), the norm $l_{p}$ for $p=1$ is transformed to the following form:

$$
l_{1}=\sum_{i \in I}\left|k_{i}-\psi_{i}\right|+\sum_{p=1}^{l}\left|\varphi_{p}-\delta_{p}\right|+\sum_{(i, j) \in U}\left|\theta_{i j}-\gamma_{i j}\right|+\sum_{p=1}^{l} \sum_{(i, j) \in U}\left|r_{i j}^{p}-t_{i j}^{p}\right|
$$




$$
=\sum_{i \in I}\left(k_{i}+\psi_{i}\right)+\sum_{p=1}^{l}\left(\varphi_{p}+\delta_{p}\right)+\sum_{(i, j) \in U}\left(\theta_{i j}+\gamma_{i j}\right)+\sum_{p=1}^{l} \sum_{(i, j) \in U}\left(r_{i j}^{p}+t_{i j}^{p}\right) .
$$

The mathematical model of the extremal problem to determine the changes parameters (5) of the problem (1)-(4) has the form:

$$
\begin{gathered}
q(k, \psi, \varphi, \delta, \theta, \gamma, r, t)=\sum_{i \in I}\left(k_{i}+\psi_{i}\right)+\sum_{p=1}^{l}\left(\varphi_{p}+\delta_{p}\right) \\
+\sum_{(i, j) \in U}\left(\theta_{i j}+\gamma_{i j}\right)+\sum_{p=1}^{l} \sum_{(i, j) \in U}\left(r_{i j}^{p}+t_{i j}^{p}\right) \longrightarrow \mathrm{min}, \\
\sum_{j \in I_{i}^{+}(U)} x_{i j}-\sum_{j \in I_{i}^{-}(U)}\left(\mu_{j i}+\theta_{j i}-\gamma_{j i}\right) x_{j i}=a_{i}+k_{i}-\psi_{i}, \\
k_{i} \geq 0, \psi_{i} \geq 0, i \in I, \\
0<\mu_{i j}+\theta_{i j}-\gamma_{i j} \leq 1, \theta_{i j} \geq 0, \gamma_{i j} \geq 0,(i, j) \in U ; \\
\sum_{(i, j) \in U}\left(\lambda_{i j}^{p}+r_{i j}^{p}-t_{i j}^{p}\right) x_{i j}=\alpha_{p}+\varphi_{p}-\delta_{p}, \quad \varphi_{p} \geq 0, \\
\delta_{p} \geq 0, p=\overline{1, l} ; r_{i j}^{p} \geq 0, t_{i j}^{p} \geq 0, \quad(i, j) \in U, p=\overline{1, l} \\
x_{i j} \geq 0, \quad(i, j) \in U .
\end{gathered}
$$

Thus, the infeasible basic solution $x=\left(x_{i j},(i, j) \in U\right)$ of the problem (1)(4) for the parameters (5) is the feasible basic solution of the extremal problem (7)-(10) for the new parameters (6), where the numerical values of increasing and decreasing for the parameters (5) are obtained by solving the extremal problem (11)-(14). Now we can use the effective methods of decomposition [5] - [8] which designed to solve network problems of linear and fractional linear mathematical programming.

\section{The Dual Problem}

The dual problem for the linear-fractional network flow programming problem (1)-(4) has the following form:

$$
g(y, r, z)=z \longrightarrow \max
$$




$$
\begin{gathered}
y_{i}-\mu_{i j} y_{j}+\sum_{p=1}^{l} \lambda_{i j}^{p} r_{p}+q_{i j} z \leq c_{i j}, \quad(i, j) \in U, \\
-\sum_{j \in I} a_{i} y_{i}-\sum_{p=1}^{l} \alpha_{p} r_{p}+\gamma z=\beta,
\end{gathered}
$$

where $z \in R^{1}, y=\left(y_{i}, i \in I\right), r=\left(r_{p}, p=\overline{1, l}\right)$.

The vector $\lambda=(y, r, z)$ is called the feasible solution of the dual problem (15) - (16), if for the components of the vector $\lambda$ are performed the restrictions (16).

Theorem 1. Let $x^{0}=\left(x_{i j}^{0},(i, j) \in U\right)$ be feasible solution of the problem (1)-(4). If for some vector $\lambda$,

$$
\lambda=(y, r, z), \quad y=\left(y_{i}, i \in I\right), r=\left(r_{p}, p=\overline{1, l}\right), \quad z \in R^{1}
$$

and for given feasible solution $x^{0}$ the following conditions are met:

$$
\begin{gathered}
y_{i}-\mu_{i j} y_{j}+\sum_{p=1}^{l} \lambda_{i j}^{p} r_{p}+q_{i j} z \leq c_{i j}, \quad(i, j) \in U, \\
-\sum_{j \in I} a_{i} y_{i}-\sum_{p=1}^{l} \alpha_{p} r_{p}+\gamma z=\beta, \\
\left(y_{i}-\mu_{i j} y_{j}+\sum_{p=1}^{l} \lambda_{i j}^{p} r_{p}+q_{i j} z-c_{i j}\right) x_{i j}^{0}=0, \quad(i, j) \in U,
\end{gathered}
$$

then $x^{0}$ is the optimal solution of the problem (1)-(4).

Proof. For the given feasible solution $x^{0}=\left(x_{i j}^{0},(i, j) \in U\right)$ of the problem (1)-(4) and for the some feasible solution $(y, r, z), y=\left(y_{i}, i \in I\right), r=\left(r_{p}, p=\right.$ $\overline{1, l}), z \in R^{l}$ of the dual problem (15)-(16), the following relations are satisfied:

$$
\left(y_{i}-\mu_{i j} y_{j}+\sum_{p=1}^{l} \lambda_{i j}^{p} r_{p}+q_{i j} z-c_{i j}\right) x_{i j}^{0}=0, \quad(i, j) \in U
$$


Then we have:

$$
\begin{aligned}
& \sum_{(i j) \in U} c_{i j} x_{i j}^{0}+\beta=\sum_{(i j) \in U}\left(y_{i}-\mu_{i j} y_{j}+\sum_{p=1}^{l} \lambda_{i j}^{p} r_{p}+q_{i j} z\right) x_{i j}^{0}+\beta \\
& =\sum_{i \in I} a_{i} y_{i}+\sum_{p=1}^{l} \alpha_{p} r_{p}+\sum_{(i j) \in U} q_{i j} z x_{i j}^{0}-\sum_{i \in I} a_{i} y_{i}-\sum_{p=1}^{l} \alpha_{p} r_{p}+y z \\
& =z \sum_{(i j) \in U} q_{i j} x_{i j}^{0}+y z=z\left(\sum_{(i j) \in U} q_{i j} x_{i j}^{0}+\gamma\right) .
\end{aligned}
$$

Thus, the objective function of the primal problem (1)-(4) coincides with the objective function for the problem (15)-(16), which is dual problem to (1)-(4):

$$
f\left(x^{0}\right)=\frac{\sum_{(i, j) \in U} c_{i j} x_{i j}^{0}+\beta}{\sum_{(i, j) \in U} q_{i j} x_{i j}^{0}+\gamma}=g(y, r, z)=z .
$$

Hence, $x^{0}=\left(x_{i j}^{0},(i, j) \in U\right)$ is the optimal solution of the problem $(1)-(4)$.

\section{The Inverse Problem}

Let $x=\left(x_{i j},(i, j) \in U\right)$ be some feasible solution of the extremal problem (1)-(4). We apply the principles of inverse optimization for minimally change of the parameters in the numerator of linear-fractional objective function (1) in order that the selected feasible solution $x$ becomes an optimal solution.

By Theorem 1, for some of vectors

$$
\lambda=(y, r, z), \lambda \in \Lambda, y=\left(y_{i}, i \in I\right), r=\left(r_{p}, p=\overline{1, l}\right), z \in R^{1},
$$

the parameters $c=\left(c_{i j},(i, j) \in U\right)$ may be replaced by such values of the parameters $\widetilde{c}=\left(\widetilde{c}_{i j},(i, j) \in U\right)$, that are carried out the conditions: $\left(y_{i}-\right.$ $\left.\mu_{i j} y_{j}+\sum_{p=1}^{l} \lambda_{i j}^{p} r_{p}+q_{i j} z-\tilde{c}_{i j}\right) x_{i j}^{0}=0,(i, j) \in U$.

Depending on the arc flows $x_{i j}^{0},(i, j) \in U$ a given feasible solution $x^{0}$ of the problem (1)-(4), we form the set of arcs $B_{1}, B_{2}$ :

$$
B_{1}=\left\{(i, j) \in U: x_{i j}^{0}=0\right\}, B_{2}=\left\{(i, j) \in U: x_{i j}^{0}>0\right\} .
$$


The inverse problem for minimally change of the parameters in the numerator of linear-fractional objective function (1) to the problem (1)-(4) has the form:

$$
\begin{gathered}
\|\widetilde{c}-c\| \longrightarrow \min , \\
y_{i}-\mu_{i j} y_{j}+\sum_{p=1}^{l} \lambda_{i j}^{p} r_{p}+q_{i j} z \leq \widetilde{c}_{i j}, \quad(i, j) \in B_{1}, \\
y_{i}-\mu_{i j} y_{j}+\sum_{p=1}^{l} \lambda_{i j}^{p} r_{p}+q_{i j} z=\widetilde{c}_{i j}, \quad(i, j) \in B_{2}, \\
-\sum_{j \in I} a_{i} y_{i}-\sum_{p=1}^{l} \alpha_{p} r_{p}+\gamma z=\beta .
\end{gathered}
$$

Let $\zeta_{i j}$ be an increase and $\eta_{i j}$ - a decrease of the parameter $c_{i j}$ of the numerator of linear-fractional objective function $(1),(i, j) \in U$.

We put $\widetilde{c}_{i j}=c_{i j}+\zeta_{i j}-\eta_{i j}, \zeta_{i j} \geq 0, \eta_{i j} \geq 0$ for all arcs $(i, j) \in U$. At the same time, $\zeta_{i j}$ and $\eta_{i j}$ can not be simultaneously receive positive values: $\zeta_{i j} \eta_{i j}=0,(i, j) \in U$.

The remaining parameters of the problem (1)-(4) do not changed. The inverse problem (18)-(19) we present as follows:

$$
\begin{gathered}
\|\widetilde{c}-c\| \longrightarrow \min , \\
y_{i}-\mu_{i j} y_{j}+\sum_{p=1}^{l} \lambda_{i j}^{p} r_{p}+q_{i j} z \leq c_{i j}+\zeta_{i j}-\eta_{i j}, \\
\zeta_{i j} \geq 0, \eta_{i j} \geq 0, \quad(i, j) \in B_{1}, \\
y_{i}-\mu_{i j} y_{j}+\sum_{p=1}^{l} \lambda_{i j}^{p} r_{p}+q_{i j} z=c_{i j}+\zeta_{i j}-\eta_{i j}, \\
\zeta_{i j} \geq 0, \eta_{i j} \geq 0, \quad(i, j) \in B_{2}, \\
-\sum_{j \in I} a_{i} y_{i}-\sum_{p=1}^{l} \alpha_{p} r_{p}+\gamma z=\beta .
\end{gathered}
$$

In accordance with the norm $l_{1}$,

$$
l_{1}=\|\widetilde{c}-c\|_{1}=\sum_{(i j) \in U}\left|\widetilde{c}_{i j}-c_{i j}\right|=\sum_{(i j) \in U}\left|\zeta_{i j}-\eta_{i j}\right|=\sum_{(i j) \in U}\left(\zeta_{i j}+\eta_{i j}\right),
$$


the mathematical model of the inverse problem for changing parameters $c_{i j}$, $(i, j) \in U$ of the objective function (1) has the form:

$$
\begin{gathered}
u(\zeta, \eta)=\sum_{(i j) \in U}\left(\zeta_{i j}+\eta_{i j}\right) \longrightarrow \min , \\
y_{i}-\mu_{i j} y_{j}+\sum_{p=1}^{l} \lambda_{i j}^{p} r_{p}+q_{i j} z \leq c_{i j}+\zeta_{i j}-\eta_{i j}, \\
\zeta_{i j} \geq 0, \eta_{i j} \geq 0, \quad(i, j) \in B_{1}, \\
y_{i}-\mu_{i j} y_{j}+\sum_{p=1}^{l} \lambda_{i j}^{p} r_{p}+q_{i j} z=c_{i j}+\zeta_{i j}-\eta_{i j}, \\
\zeta_{i j} \geq 0, \eta_{i j} \geq 0, \quad(i, j) \in B_{2}, \\
-\sum_{j \in I} a_{i} y_{i}-\sum_{p=1}^{l} \alpha_{p} r_{p}+\gamma z=\beta,
\end{gathered}
$$

where $\zeta=\left(\zeta_{i j},(i, j) \in U\right), \eta=\left(\eta_{i j},(i, j) \in U\right)$.

As a result of solving the inverse problem (22)-(23) we obtained the values $\zeta_{i j}, \eta_{i j}$ increasing and decreasing respectively for each parameter $c_{i j}, \zeta_{i j} \geq 0$, $\eta_{i j} \geq 0,(i, j) \in U$. The new parameters $\widetilde{c}_{i j}=c_{i j}+\zeta_{i j}-\eta_{i j},(i, j) \in U$ minimally different from the initial values of the parameters $c_{i j},(i, j) \in U$. The feasible solution $x^{0} \in X$ of the problem (1)-(4) is the optimal solution for the following extremal problem:

$$
\begin{aligned}
& \frac{\sum_{(i, j) \in U}\left(c_{i j}+\zeta_{i j}-\eta_{i j}\right) x_{i j}+\beta}{\sum_{(i, j) \in U} q_{i j} x_{i j}+\gamma} \longrightarrow \min \\
& \sum_{j \in I_{i}^{+}(U)} x_{i j}-\sum_{j \in I_{i}^{-}(U)} \mu_{j i} x_{j i}=a_{i}, \quad i \in I, \\
& \sum_{(i, j) \in U} \lambda_{i j}^{p} x_{i j}=\alpha_{p}, p=\overline{1, l}, x_{i j} \geq 0, \quad(i, j) \in U \text {. }
\end{aligned}
$$




\section{References}

[1] D. Burton, P.L. Toint, On an instance of the inverse shortest paths problem, J. Math. Programming, 53 (1992), 45-61.

[2] R.K. Ahuja, J.B. Orlin, Inverse optimization, Operation Research, 49, Issue 5 (2001), 771-783.

[3] L.A. Pilipchuk, A.S. Pilipchuk, Modeling parameters of the lower and upper bounds and parameters of the objective function for generalized network flow programming problems, In: AIP Conf. Proc., American Institute of Physics, 1690, 060007 (2015), 10 pp., doi: 10.1063/1.4936745.

[4] L.A. Pilipchuk, Obratnaya zadacha korrektirovki parametrov ogranichenii dlya odnoi lineinoi neodnorodnoi zadachi setevoi optimizacii, Vestnik BGU, Ser. 1, 1 (2016), 136143, In Russian.

[5] L.A. Pilipchuk, Primenenie konstruktivnih metodov dekompozicii dlya resheniya odnoi nelineinoi zadachi setevoi optimizacii, Vesnik Grodzenskaga Dzyarjaynaga Yniversiteta imya Yanki Kupali, Ser. 2, 6, No. 2 (192) (2015), 54-61, In Russian.

[6] L.A. Pilipchuk, Lineinie neodnorodnie zadachi potokovogo programmirovaniya, Minsk: BGU (2009), In Russian.

[7] R. Gabasov, F.M. Kirillova, Metodi Lineinogo Programmirovaniya, Volume 3 Chastyah, Chast 3. Specialnie Zadachi, Minsk, Belorussian State Univ. (1980), In Russian.

[8] L.A. Pilipchuk, Linear-fractional programming: problems of optimization of inhomogeneous flows in the generalized networks, Intern. J. Pure and Appl. Math., 82, No 2 (2013), 261-273. 